\title{
Mutual Coupling Compensation for Direction-of-Arrival Estimations Using the Receiving-Mutual-Impedance Method
}

\author{
Hoi Shun Lui ${ }^{1}$ and Hon Tat Hui ${ }^{2}$ \\ ${ }^{1}$ Department of Signals and Systems, Chalmers University of Technology, 41296 Gothenburg, Sweden \\ ${ }^{2}$ Department of Electrical and Computer Engineering, National University of Singapore, Singapore, 117576
}

Correspondence should be addressed to Hoi Shun Lui, antony.lui@chalmers.se

Received 25 November 2009; Accepted 8 January 2010

Academic Editor: Marek E. Bialkowski

Copyright (C) 2010 H. S. Lui and H. T. Hui. This is an open access article distributed under the Creative Commons Attribution License, which permits unrestricted use, distribution, and reproduction in any medium, provided the original work is properly cited.

\begin{abstract}
A short review of the receiving-mutual-impedance method (RMIM) for mutual coupling compensation in direction finding applications using linear array is conducted. The differences between the conventional-mutual-impedance method (CMIM) and RMIM, as well as the three different determination methods for receiving mutual impedance (RMI), will be discussed in details. As an example, direction finding with better accuracies is used for demonstrating the superiority of mutual coupling compensation using RMIM.
\end{abstract}

\section{Introduction}

Signal processing algorithms of most antenna array applications usually assume that interactions of signals and antenna elements are independent to each other. Such assumption is not necessary to be true especially when the antenna elements are closely spaced. For transmitting applications, the transmitted signals from an antenna element would be received by the other elements in the array and reradiate. The entire transmitted signal is not just the sum of the individual signals from each antenna element, but also the reradiate field from each antenna element. Similarly in receiving applications, electromagnetic wave is first received and current is then induced on an antenna element. The induced current on the antenna element reradiates electromagnetic field which would be received by other elements on the array. The wavefront of the incoming signal is distorted by the reradiated field. In both cases, interactions between the antenna elements distorted the wavefront of the signals. Such effect is known as mutual coupling and it is usually considered as a defect which degrades the performance of array signal processing algorithms.
Existence of the mutual coupling effect in antenna array has been well known throughout the years and extensive work has already been contributed to either reducing or compensating such undesirable effects. Gupta and Ksienski [1] used a circuit theory approach based on the use of the conventional mutual impedance (CMI) [2] to analyze the effect of antenna mutual coupling. This method, namely the conventional-mutual-impedance method (CMIM), has become the most popular method for mutual coupling analysis as CMIs can easily be measured directly or obtained indirectly from the measurement of the S-parameters. Later, Yeh et al. [3] applied the CMIM to decouple the voltages measured from the antenna terminals of a receiving antenna array in a Direction-of-Arrival (DOA) estimation application. The results with better accuracies of DOA estimation demonstrate that the undesirable mutual coupling effect can be partially compensated via a decoupling process with CMI.

Throughout the last two decades, significant amount of interest has been contributed to mutual coupling compensation in DOA estimations and adaptive nulling. Various methods such as calibration methods [4-6], full-wave method [7-9], and receiving-mutual-impedance method 
(RMIM) [10-15] have been proposed and demonstrate better mutual coupling compensation performance over the CMIM method. A recent review of various mutual coupling methods can be found in [16]. Direct comparisons of these methods for the same DOA estimation problem can be found in [17]. The superiority of these methods over the CMIM relies on the fact that the mutual coupling of the receiving array is considered, as compared to the CMIM which the CMIs are determined when the array is in transmitting mode. The differences of the mutual coupling problems for transmitting and receiving arrays have been covered in Balanis [18] and Daniel [19]. The differences between the CMIM, RMIM, full-wave method and calibration method have recently been discussed in Lui et al. [20].

In this paper, a review of the RMIM in the context of DOA estimation using linear array is presented. The concept of receiving mutual impedance (RMI) was first introduced by Hui $[10,11]$ and applied on uniform linear array (ULA) for DOA estimations and adaptive nulling. A review of the RMIM will be given in details in the next section. In particular, the formation of RMIM, the "singlemode" approximation [10] as well as the difference between RMIM and CMIM will be discussed in details. Throughout the years, there have been three methods to determine the RMI for linear arrays [12, 13, 15]. Discussions of these determination methods will be given in details in Section 3 . A DOA estimation example using Matrix Pencil Method (MPM) $[9,21,22]$ with better accuracies demonstrate the superiority of mutual coupling compensation of RMIM.

\section{Receiving Mutual Impedance}

Consider a simple case of a two-element dipole array. According to the standard MoM analysis, the entire electromagnetic problem of the antenna array with each antenna discretized into $M$ segments can be written as [10,23]

$$
\left[\begin{array}{ll}
{\left[Z_{11}\right]} & {\left[Z_{12}\right]} \\
{\left[Z_{21}\right]} & {\left[Z_{22}\right]}
\end{array}\right]\left[\begin{array}{l}
{\left[I_{1}\right]} \\
{\left[I_{2}\right]}
\end{array}\right]=\left[\begin{array}{l}
{\left[V_{1}\right]} \\
{\left[V_{2}\right]}
\end{array}\right],
$$

where the matrix blocks are

$$
\begin{gathered}
{\left[Z_{\alpha \beta}\right]=\left[\begin{array}{cccc}
z_{11}^{\alpha \beta}-Z_{L} & z_{12}^{\alpha \beta} & \cdots & z_{1 M}^{\alpha \beta} \\
z_{21}^{\alpha \beta} & z_{22}^{\alpha \beta} & \cdots & z_{2 M}^{\alpha \beta} \\
\vdots & \vdots & \ddots & \vdots \\
z_{M 1}^{\alpha \beta} & z_{M 2}^{\alpha \beta} & \cdots & z_{M M}^{\alpha \beta}
\end{array}\right],} \\
{\left[I_{\alpha}\right]=\left[\begin{array}{c}
I_{1}^{\alpha} \\
I_{2}^{\alpha} \\
\vdots \\
I_{M}^{\alpha}
\end{array}\right],}
\end{gathered}
$$

respectively. $\alpha$ and $\beta$ ranges from 1 and 2 for the case of two antenna elements. The elements in the $\left\lfloor Z_{\alpha \beta}\right\rfloor$ matrix, $z_{a b}^{\alpha \beta}$, correspond to the impedance between the segment $a$ of antenna $\alpha$ and segment $b$ of antenna $\beta, Z_{L}$ corresponds to the load impedance of the antenna terminal. The elements $I_{i}^{\alpha}$ and $V_{i}^{\alpha}$ of the $\left[I_{\alpha}\right]$ and $\left[V_{\alpha}\right]$ matrices, respectively are the induced current and the excitation voltage to the corresponding $i$ th segment of the antenna $\alpha$, where $m=1,2, \ldots, M$. Expanding from (1), this gives

$$
\begin{aligned}
& {\left[\begin{array}{cccc}
z_{11}^{11}-Z_{L} & z_{12}^{11} & \cdots & z_{1 M}^{11} \\
z_{21}^{11} & z_{22}^{11} & \cdots & z_{2 M}^{11} \\
\vdots & \vdots & \ddots & \vdots \\
z_{M 1}^{11} & z_{M 2}^{11} & \cdots & z_{M M}^{11}
\end{array}\right]\left[\begin{array}{c}
I_{1}^{1} \\
I_{2}^{1} \\
\vdots \\
I_{M}^{1}
\end{array}\right]} \\
& +\left[\begin{array}{cccc}
z_{11}^{12}-Z_{L} & z_{12}^{12} & \cdots & z_{1 M}^{12} \\
z_{21}^{12} & z_{22}^{12} & \cdots & z_{2 M}^{12} \\
\vdots & \vdots & \ddots & \vdots \\
z_{M 1}^{12} & z_{M 2}^{12} & \cdots & z_{M M}^{12}
\end{array}\right]\left[\begin{array}{c}
I_{1}^{2} \\
I_{2}^{2} \\
\vdots \\
I_{M}^{2}
\end{array}\right]=\left[\begin{array}{c}
V_{1}^{1} \\
V_{2}^{1} \\
\vdots \\
V_{M}^{1}
\end{array}\right] \text {, } \\
& {\left[\begin{array}{cccc}
z_{11}^{21}-Z_{L} & z_{12}^{21} & \cdots & z_{1 M}^{21} \\
z_{21}^{21} & z_{22}^{21} & \cdots & z_{2 M}^{21} \\
\vdots & \vdots & \ddots & \vdots \\
z_{M 1}^{21} & z_{M 2}^{21} & \cdots & z_{M M}^{21}
\end{array}\right]\left[\begin{array}{c}
I_{1}^{1} \\
I_{2}^{1} \\
\vdots \\
I_{M}^{1}
\end{array}\right]} \\
& +\left[\begin{array}{cccc}
z_{11}^{22}-Z_{L} & z_{12}^{22} & \cdots & z_{1 M}^{22} \\
z_{21}^{22} & z_{22}^{22} & \cdots & z_{2 M}^{22} \\
\vdots & \vdots & \ddots & \vdots \\
z_{M 1}^{22} & z_{M 2}^{22} & \cdots & z_{M M}^{22}
\end{array}\right]\left[\begin{array}{c}
I_{1}^{2} \\
I_{2}^{2} \\
\vdots \\
I_{M}^{2}
\end{array}\right]=\left[\begin{array}{c}
V_{1}^{2} \\
V_{2}^{2} \\
\vdots \\
V_{M}^{2}
\end{array}\right] .
\end{aligned}
$$

As we can see, the mutual coupling effect is due to the off-diagonal matrix blocks, that is, $\left\lfloor Z_{\alpha \beta}\right\rfloor$ with $\alpha \neq \beta$. If the entire current distribution of the antenna $\left[I_{\alpha}\right]$ and the full knowledge of the all elements in the impedance matrices $\left\lfloor Z_{\alpha \beta}\right\rfloor$ are known, the mutual coupling effect can be accurately quantified and removed [7-9]. However, this can only be done in the modeling domain. In practice, it is not possible to measure the entire current distribution of the antenna, that is, all the values in the $\left[I_{\alpha}\right]$ matrix. Only the terminal voltages of the antenna terminals, that is, $V_{t}^{\alpha}=I_{1}^{\alpha} Z_{L}$ can be measured. As a result, Hui [10] has introduced the RMI based on the "single-mode" assumption, that is, we treat the current induced on the each antenna element of the array consist of a single-mode current based on the current at the antenna terminal. For a two-element receiving array, this can be written as

$$
\begin{aligned}
& U_{1}+Z_{t}^{12} I_{2}=V_{1}, \\
& U_{2}+Z_{t}^{21} I_{1}=V_{2},
\end{aligned}
$$

where $Z_{t}^{12}$ and $Z_{t}^{21}$ are defined as the RMI between the two antennas, $I_{1}=I_{1}^{1}$ and $I_{2}=I_{1}^{2}$ are the induced current in the antenna terminals, $V_{1}$ and $V_{2}$ are the induced voltages 
in the antenna terminal 1 and 2, respectively, and $U_{1}$ and $U_{2}$ are the terminal voltages solely based on the incoming signals without any mutual coupling effect. The subscript $t$ denotes that the RMI is defined based on the current and voltage parameters at the antenna terminal.

It is apparent that under the "single-mode" assumption, the RMIs defined in (4a) and (4b) are no longer the elements of the $\left\lfloor Z_{\alpha \beta}\right\rfloor$ matrix in the original integral equation formulation in (1). As shown in (4a) and (4b), the RMI varies as the induced terminal current $I_{t}^{\alpha}$ and voltages $V_{t}^{\alpha}$ of the antennas, as well as the load impedance (if $Z_{L}$ in (3) changes, the entire electromagnetic problem changes and so as the RMI). For omnidirectional antenna elements, the current distribution remains to be the same for any angle of azimuth angle $\phi$ in the elevation plane of $\theta=90^{\circ}$ (horizontal plane) [13].

Now we extend the concept to antenna array with $N$ antenna elements and each of them are terminated with the same load impedance $Z_{L}$. The measured voltage at antenna terminal $V_{k}$ can be written as

$$
V_{k}=Z_{L} I_{k}=U_{k}+W_{k}
$$

where

$$
\begin{aligned}
W_{k}= & Z_{t}^{k, 1} I_{1}+Z_{t}^{k, 2} I_{2}+\cdots+Z_{t}^{k, k-1} I_{k-1}+Z_{t}^{k, k+1} I_{k+1}+\cdots \\
& +Z_{t}^{k, N} I_{N} .
\end{aligned}
$$

Once again $U_{k}$ is the measured terminal voltage due to the incoming signal alone, $W_{k}$ is the voltage due to the mutual coupling effect from other array elements, $Z_{t}^{k, i}$ is the RMI between antenna elements $k$ and $i, I_{i}$ is the current induced at the antenna terminal, given by $I_{i}=V_{i} / Z_{L}$ for $i=1,2, \ldots, N$. Rearranging (5a) and (5b) the relationship between $U_{k} s$ and $V_{k} s$ can be written as

$$
\left.\begin{array}{ccccc}
1 & -\frac{Z_{t}^{1,2}}{Z_{L}} & \cdots & -\frac{Z_{t}^{1, N-1}}{Z_{L}} & -\frac{Z_{t}^{1, N}}{Z_{L}} \\
-\frac{Z_{t}^{2,1}}{Z_{L}} & 1 & \cdots & -\frac{Z_{t}^{2, N-1}}{Z_{L}} & -\frac{Z_{t}^{2, N}}{Z_{L}} \\
\vdots & \vdots & \ddots & \vdots & \vdots \\
-\frac{Z_{t}^{N-1,1}}{Z_{L}} & -\frac{Z_{t}^{N-1,2}}{Z_{L}} & \cdots & 1 & -\frac{Z_{t}^{N-1, N}}{Z_{L}} \\
-\frac{Z_{t}^{N, 1}}{Z_{L}} & -\frac{Z_{t}^{N, 2}}{Z_{L}} & \cdots & -\frac{Z_{t}^{N, N-1}}{Z_{L}} \\
V_{1} \\
V_{2} \\
\vdots \\
U_{1} \\
U_{N-1} \\
V_{N}
\end{array}\right]_{N \times 1}\left[\begin{array}{c}
\vdots \\
U_{N-1} \\
U_{N}
\end{array}\right]_{N \times 1}
$$

With a priori knowledge of the RMIs together with the terminal voltages $V_{k}$ s from the DOA problem, the uncoupled voltages $U_{k} s$ can thus be determined using (6) and the undesirable mutual coupling effect is thus compensated.

\section{Comparision with the Conventional-Mutual-Impedence Method}

Other than the RMIM, another well establishing method, the CMIM has been well known in the literature. The differences between the two methods have not been well discussed and we take this opportunity to give a clear explanation. According to [1], the relationship between the voltages at terminal $k$ with other antenna terminals can be written as

$$
\begin{aligned}
V_{1}= & I_{1} Z_{k, 1}+I_{2} Z_{k, 2}+\cdots+I_{i} Z_{k, i}+\cdots+I_{k} Z_{k, k}+\cdots \\
& +I_{N} Z_{k, N}+V_{O k},
\end{aligned}
$$

where $Z_{k, i}$ is the CMI between antenna terminals $k$ and $j, Z_{k, k}$ corresponds to the self impedance of the antenna element $k, I_{i}$ corresponds to the induced current at the antenna terminal $i$ and $V_{O k}$ is defined as the open-circuit voltage at terminal $k$ when all other antenna elements under opencircuit condition, that is, $I_{i}=0$ for $i=1,2, \ldots, N$. The relationship between the terminal voltage and current can be given by

$$
I_{1}^{t}=-\frac{V_{i}^{t}}{Z_{L}},
$$

where $Z_{L}$ is the load impedance terminated at the feed. With the open-circuit condition and substituting (8) into (7), the relationship between the open-circuit voltages and terminal voltages can be written as

$$
\begin{gathered}
{\left[\begin{array}{cccc}
1+\frac{Z_{11}}{Z_{L}} & \frac{Z_{12}}{Z_{L}} & \cdots & \frac{Z_{1 N}}{Z_{L}} \\
\frac{Z_{21}}{Z_{L}} & 1+\frac{Z_{22}}{Z_{L}} & \cdots & \frac{Z_{2 N}}{Z_{L}} \\
\vdots & \vdots & \ddots & \vdots \\
\frac{Z_{N 1}}{Z_{L}} & \frac{Z_{N 2}}{Z_{L}} & \cdots & 1+\frac{Z_{N N}}{Z_{L}}
\end{array}\right]_{N \times N}\left[\begin{array}{c}
V_{1} \\
V_{2} \\
\vdots \\
V_{N}
\end{array}\right]_{1 \times N}} \\
=\left[\begin{array}{c}
V_{O 1} \\
V_{O 2} \\
\vdots \\
V_{O N}
\end{array}\right]_{1 \times N} .
\end{gathered}
$$

Comparing CMIM and RMIM, there are three main differences between the two. They are:

(1) The CMI is determined when the antenna array is in transmitting mode while the RMI is determined when the array is in receiving mode. As discussed in [18-20], the mutual coupling problems of transmitting and receiving arrays are two different problems in general and they should be treated separately. 
(2) The CMI is defined when the antenna element is open circuit while the RMI is defined when the antenna is loaded. The current distribution of the antenna varies with different load impedance. The antenna is usually terminated with load impedance in practice. Strictly speaking, an open-circuit assumption provides only an approximate solution to the mutual coupling characterization.

(3) The two mutual impedances are defined differently and thus the decoupling matrices are different $((6)$ and (9)). In particular, notice that the knowledge of self-impedance is not required for the RMIM.

\section{Determination Methods of the Receiving Mutual Impedance}

A discussion of the three determination methods of the RMI is covered. The motivations, differences and limitations between these methods are discussed in details.

4.1. Method 1. In this method, the RMI is determined by first taking the current distribution of an antenna element under plane-wave excitation. Here, a two-element dipole array is considered and the incident wave is coming from the elevation plane of $\theta=90^{\circ}$ (perpendicular to the dipole element). Analytical expression of the current distribution can be found in the literature [10]. In the computational domain, such current distribution is then used to excite an antenna element in the array and the coupled induced terminal currents $I_{i}$ and voltages $V_{i}$ in other antenna elements can then be obtained. The terminal voltages that are solely under plane wave excitation $U_{i}$ are determined by considering the current distribution of an antenna element with antenna $k$ removed. The RMIs of the two antenna elements $k$ and $i$ can be calculated by rearranging (4a) or (4b) as

$$
Z_{t}^{k, i}=\frac{V_{k}-U_{k}}{I_{i}}=\frac{V_{k}-U_{k}}{V_{i}} Z_{L} .
$$

The limitation of this method is that the accuracy of the determined RMI is strongly dependant on the accuracies of the approximated current distribution. In practice, the current distribution varies when are more than one signal or when the incoming elevation angle deviates from $\theta=90^{\circ}$. Furthermore, another limitation is that the RMI can only be determined via numerical modeling. Alternative solution is thus required if one would like to measure the RMI.

4.2. Method 2. In this method $[12,13]$, the two antenna elements $k$ and $i$ are first illuminated by plane-wave excitation and the terminal voltages $V_{i}$ and $V_{k}$ are obtained. Next, antenna element $i$ is removed from the array and the terminal voltage of antenna $k$ that is solely under plane wave excitation, $U_{k}$, are thus obtained. The RMI, $Z_{t}^{k, i}$, can then be determined using (10). The study in [12] has shown that the RMI can be measured experimentally and the results are comparable to the numerical modeling using MoM. This method provides an accurate solution as it does not require any assumption of the current distribution. Furthermore, the RMI can be obtained experimentally.
The main drawback of both method 1 and 2 is that only two antenna elements are considered at a time and the scattering effect from other elements (if there are more than two elements in the array) are not taken into account. According to (5a) and (5b), the mutual coupling problem for a $N$-element receiving array can be written as

$$
\begin{aligned}
V_{k}= & U_{k}+Z_{t}^{k, 1} I_{1}+Z_{t}^{k, 2} I_{2}+\cdots+Z_{t}^{k, k-1} I_{k-1}+Z_{t}^{k, k+1} I_{k+1} \\
& +\cdots+Z_{t}^{k, N} I_{N} .
\end{aligned}
$$

Consider all the voltages and current values are found as described above, there are $N-1$ unknowns in (11) $(N-1$ RMIs) which cannot be solved mathematically. As a result, only two antennas can be considered in one time in the previous two methods and removal of "other" antenna elements is needed when $N>2$. Strictly speaking, this method only provides an accurate solution for the case of two antenna elements. When there are more than two antennas, the "extra" antennas have to be removed and thus the electromagnetic problem has changed. The scattering effect from "other elements" has not been taken into consideration and thus alternative solution is required to handle such drawback.

4.3. Method 3. For antenna arrays with omnidirectional antenna elements, the RMI remains to be a constant as the azimuth angle $\phi$ varies. Consider the terminal voltages are measured at $\phi_{n}$ and repeat the measurement at $N-1$ incoming aspects, (11) can be written as

$$
\begin{aligned}
& {\left[\begin{array}{c}
V_{k}^{\phi_{1}}-U_{k}^{\phi_{1}} \\
V_{k}^{\phi_{2}}-U_{k}^{\phi_{2}} \\
\vdots \\
V_{k}^{\phi_{N-2}}-U_{k}^{\phi_{N-2}} \\
V_{k}^{\phi_{N-1}}-U_{k}^{\phi_{N-1}}
\end{array}\right]_{(N-1) \times 1}} \\
& =\left[\begin{array}{cccccc}
I_{1}^{\phi_{1}} & \cdots & I_{k-1}^{\phi_{1}} & I_{k+1}^{\phi_{1}} & \cdots & I_{N}^{\phi_{1}} \\
I_{1}^{\phi_{2}} & \cdots & I_{k-1}^{\phi_{2}} & I_{k+1}^{\phi_{2}} & \cdots & I_{N}^{\phi_{2}} \\
\vdots & \ddots & \vdots & \vdots & \ddots & \vdots \\
I_{1}^{\phi_{N-2}} & \cdots & I_{k-1}^{\phi_{N-2}} & I_{k+1}^{\phi_{N-2}} & \cdots & I_{N}^{\phi_{N-2}} \\
I_{1}^{\phi_{N-1}} & \cdots & I_{k-1}^{\phi_{N-1}} & I_{k+1}^{\phi_{N-1}} & \cdots & I_{N}^{\phi_{N-1}}
\end{array}\right]_{(N-1) \times(N-1)} \\
& \times\left[\begin{array}{c}
Z_{t}^{k, 1} \\
\vdots \\
Z_{t}^{k, k-1} \\
Z_{t}^{k, k+1} \\
\vdots \\
Z_{t}^{k, N-1}
\end{array}\right]_{(N-1) \times 1}
\end{aligned}
$$


TABLE 1: DOA estimation using MPM using a ULA with 8 antenna elements with the element separation of $\Delta=0.3 \lambda$. DOA estimation is based on the decoupled voltages using RMI determined based on methods 2 and 3 .

\begin{tabular}{|c|c|c|c|c|c|}
\hline & Direction & Mean & Bias & Standard Dev. & RMSE \\
\hline \multirow{3}{*}{ Method 2} & $20^{\circ}$ & $19.6199^{\circ}$ & $0.3801^{\circ}$ & $0.0938^{\circ}$ & $0.3915^{\circ}$ \\
\hline & $35^{\circ}$ & $34.6402^{\circ}$ & $0.3598^{\circ}$ & $0.6327^{\circ}$ & $0.7276^{\circ}$ \\
\hline & $100^{\circ}$ & $100.0012^{\circ}$ & $0.0012^{\circ}$ & $0.0102^{\circ}$ & $0.0102^{\circ}$ \\
\hline \multirow{3}{*}{ Method 3} & $20^{\circ}$ & $19.8486^{\circ}$ & $0.1514^{\circ}$ & $0.0210^{\circ}$ & $0.1529^{\circ}$ \\
\hline & $35^{\circ}$ & $34.8913^{\circ}$ & $0.1087^{\circ}$ & $0.1068^{\circ}$ & $0.1524^{\circ}$ \\
\hline & $100^{\circ}$ & $100.0068^{\circ}$ & $0.0068^{\circ}$ & $0.0015^{\circ}$ & $0.0069^{\circ}$ \\
\hline
\end{tabular}

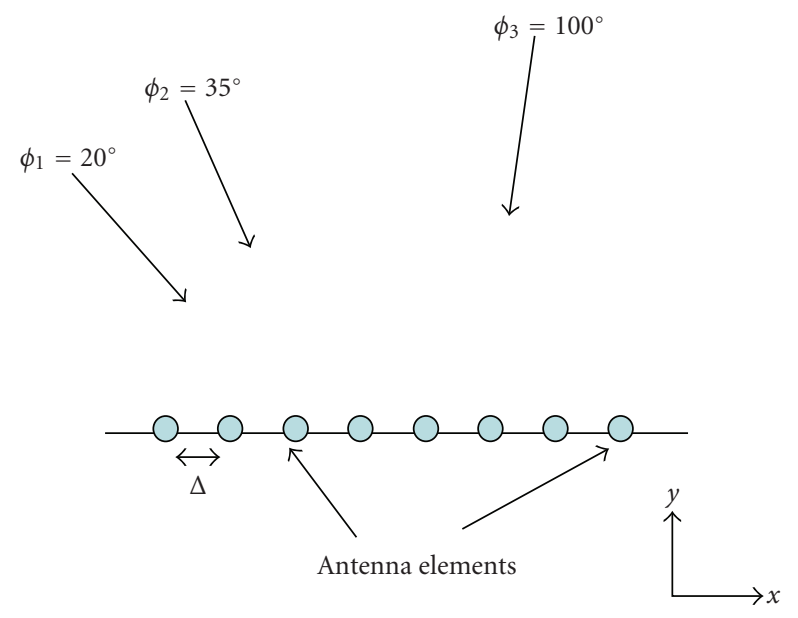

FIGURE 1: The top view of the DOA estimation problems. The ULA is lined in the $x$ axis and the incoming signals are coming from the azimuth angles of $\phi_{1}=20^{\circ}, \phi_{2}=35^{\circ}$ and $\phi_{3}=100^{\circ}$. The antenna separation is denoted as $\Delta$.

where the superscripts $\phi_{n}$ on the voltages and currents terms correspond to the voltages and currents measured under the plane-wave illumination from the azimuth angle of $\phi_{n}$, respectively. The RMI $Z_{t}^{k, i}$ s can be determined by solving (11), where $i=1,2, \ldots, k-1, k+1, \ldots, N$.

As previously mentioned, the first advantage this method does not require the removal of antenna elements such that the scattering effect from all antenna elements is taken into consideration in the calculation when we calculate/measure $V_{k}$. Preliminary results in [15] have first demonstrated that better accuracies can be obtained for DOA estimations using the RMI determined based on method 3 as compared to method 2. More importantly, as the element separation of the antenna elements becomes smaller, the scattering effect from other elements becomes significant and RMI determined using method 2 may fail to compensate the mutual coupling effect.

To demonstrate the superiority of method 3 , a DOA estimation example using two 8-element uniform linear arrays with element separations of $0.3 \lambda$ and $0.15 \lambda$ is considered. Decoupling using CMIM is not included here as it has previously been demonstrated $[10,11,14,15]$ that RMIM is better than CMIM and we are not going to repeat it here

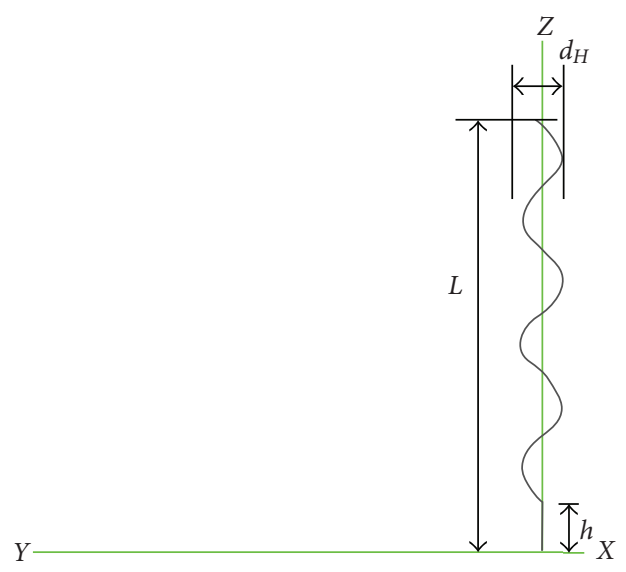

Figure 2: The NMHA with $L=25 \mathrm{~mm}, d_{H}=2.6 \mathrm{~mm}, h=3 \mathrm{~mm}$ and $d_{w}=0.6 \mathrm{~mm}$.

again. Normal-Mode Helical Antenna (NMHA) element shown in Figure 2 is considered. Three signals coming from the azimuth directions of $\phi_{1}=20^{\circ}, \phi_{2}=35^{\circ}$, and $\phi_{3}=100^{\circ}$ (all from the elevation angle of $\theta=90^{\circ}$ ) shown in Figure 1 are considered. The incoming signals are contaminated with Additive Gaussian White Noise (AGWN) with a Signal-toNoise Ratio (SNR) of $10 \mathrm{~dB}$. The entire simulations are done in full-wave electromagnetic solver FEKO [24] so that the scattering effect from all antenna elements is taken into account. The signals are first compensated using the RMIM based on the RMI determined using method 2 and a Matrix Pencil Method (MPM) algorithm is used for DOA estimations. The results out of 1000 simulations are tabulated in Tables 1 and 2. For the case with an element separation of $0.3 \lambda$, there is not significant differences for the estimated incoming directions when the RMI is determined using the two methods. However, as the element separation is reduced to $0.15 \lambda$, as shown in Table 2 , it is found that the estimated directions of signal 1 and signal 2 becomes $26^{\circ}$ and $80^{\circ}$, respectively, which are not correct. Using the RMI determined using method 3 , three incoming signals can be clearly resolved with less than $0.5^{\circ}$ of bias, standard deviations, root-mean-square errors (RMSEs).

Further investigations about the solution of the RMI determined using different number of incidence, can be found in our recent publication [25]. The only limitation of 
TABLE 2: DOA estimation using MPM and a ULA with 8 antenna elements with the element separation of $\Delta=0.15 \lambda$. DOA estimation is based on the decoupled voltages using the RMI determined based on methods 2 and 3 .

\begin{tabular}{|c|c|c|c|c|c|}
\hline & Direction & Mean & Bias & Standard dev. & RMSE \\
\hline \multirow{3}{*}{ Method 2} & $20^{\circ}$ & $26.8300^{\circ}$ & $6.8300^{\circ}$ & $1.9839^{\circ}$ & $7.1121^{\circ}$ \\
\hline & $35^{\circ}$ & $79.8916^{\circ}$ & $44.8916^{\circ}$ & $8.9505^{\circ}$ & $45.7743^{\circ}$ \\
\hline & $100^{\circ}$ & $99.3991^{\circ}$ & $0.6008^{\circ}$ & $3.4001^{\circ}$ & $3.4511^{\circ}$ \\
\hline \multirow{3}{*}{ Method 3} & $20^{\circ}$ & $20.1861^{\circ}$ & $0.1861^{\circ}$ & $0.4111^{\circ}$ & $0.4511^{\circ}$ \\
\hline & $35^{\circ}$ & $35.1614^{\circ}$ & $0.1614^{\circ}$ & $0.4504^{\circ}$ & $0.4783^{\circ}$ \\
\hline & $100^{\circ}$ & $100.0078^{\circ}$ & $0.0078^{\circ}$ & $0.0078^{\circ}$ & $0.0110^{\circ}$ \\
\hline
\end{tabular}

this method is that it is only applicable to omnidirectional antenna elements. Alternative solutions are thus required for nonomnidirectional antenna elements $[26,27]$.

\section{Conclusions}

A review of the RMIM for mutual coupling compensation in the scope of DOA estimations using ULA is presented. In particular, the definition of RMI, the "single-mode" approximation, as well as the differences between CMIM and RMIM are discussed in details. The advantages and limitations of the three determination methods of RMI have also covered followed by a DOA estimation example which demonstrates the feasibilities and superiorities of mutual coupling compensation using RMI. In addition to theoretical studies, we have recently demonstrated experimentally that the RMIM can be used for mutual coupling compensation [28].

The RMIM has also been applied to mutual coupling compensation of DOA estimations using circular array [29] and phase array in Magnetic Resonance Imaging (MRI) [30-32]. Recently, the RMI has been adopted to model the mutual coupling effect of Multiple-Input Multiple-Output (MIMO) system and new models for channel capacities of the MIMO systems have been developed $[33,34]$. It is no doubt that the RMIM is an important model for mutual coupling characterization and it is not difficult to see that such model can be used in other radar, biomedical, and communication applications.

\section{Acknowledgments}

This work was supported in part by the National University of Singapore (NUS) under Grant no. R-263-000-469-112 and in part by the US ONR research fund under the Project no. PR. NO. 09PR03332-01.

\section{References}

[1] I. J. Gupta and A. A. Ksienski, "EEffect of mutual coupling on the performance of adaptive arrays," IEEE Transactions on Antennas and Propagation, vol. 31, no. 5, pp. 785-791, 1983.

[2] R. E. Collin and F. J. Zucker, Eds., Antenna Theory, McGrawHill, New York, NY, USA, 1968.

[3] C.-C. Yeh, M.-L. Leou, and D. R. Ucci, "Bearing estimations with mutual coupling present," IEEE Transactions on Antennas and Propagation, vol. 37, no. 10, pp. 1332-1335, 1989.
[4] B. Friedlander and A. J. Weiss, "Direction finding in the presence of mutual coupling," IEEE Transactions on Antennas and Propagation, vol. 39, no. 3, pp. 273-284, 1991.

[5] K. R. Dandekar, H. Ling, and G. Xu, "Experimental study of mutual coupling compensation in smart antenna applications," IEEE Transactions on Wireless Communications, vol. 1, no. 3, pp. 480-487, 2002.

[6] R. Hossa and M. Bialkowski, "Mutual coupling compensation in narrowband small linear-antenna arrays," Microwave and Optical Technology Letters, vol. 40, no. 5, pp. 391-396, 2004.

[7] K. M. Pasala and E. M. Friel, "Mutual coupling effects and their reduction in wideband direction of arrival estimation," IEEE Transactions on Aerospace and Electronic Systems, vol. 30, no. 4, pp. 1116-1122, 1994.

[8] R. S. Adve and T. K. Sarkar, "Compensation for the effects of mutual coupling on direct data domain adaptive algorithm," IEEE Transactions on Antennas and Propagation, vol. 48, no. 1 , pp. 86-94, 2000.

[9] C. K. E. Lau, R. S. Adve, and T. K. Sarkar, "Minimum norm mutual coupling compensation with applications in direction of arrival estimation," IEEE Transactions on Antennas and Propagation, vol. 52, no. 8, pp. 2034-2041, 2004.

[10] H. T. Hui, "A practical approach to compensate for the mutual coupling effect in an adaptive dipole array," IEEE Transactions on Antennas and Propagation, vol. 52, no. 5, pp. 1262-1269, 2004.

[11] H. T. Hui, "Improved compensation for the mutual coupling effect in a dipole array for direction finding," IEEE Transactions on Antennas and Propagation, vol. 51, no. 9, pp. 2498-2503, 2003.

[12] H. T. Hui, "A new definition of mutual impedance for application in dipole receiving antenna arrays," IEEE Antennas \& Wireless Propagation Letters, vol. 3, no. 1, pp. 364-367, 2004.

[13] H. T. Hui, H. P. Low, T. T. Zhang, and Y. L. Lu, "Receiving mutual impedance between two normal-mode helical antennas (NMHAs)," IEEE Antennas and Propagation Magazine, vol. 48, no. 4, pp. 92-96, 2006.

[14] T. T. Zhang, H. T. Hui, and Y. L. Lu, "Compensation for the mutual coupling effect in the ESPRIT direction finding algorithm by using a more effective method," IEEE Transactions on Antennas and Propagation, vol. 53, no. 4, pp. 1552-1555, 2005.

[15] H.-S. Lui, H. T. Hui, and M. S. Leong, "A new calculation method of the receiving mutual impedance for linear antenna array," in Proceedings of Asia Pacific Microwave Conference (APMC '08), Macau, China, December 2008.

[16] H. T. Hui, "Decoupling methods for the mutual coupling effect in antenna arrays: a review," Recent Patents on Engineering, vol. 1, pp. 187-193, 2007. 
[17] Y. Wu and Z. Nie, "On the improvement of the mutual coupling compensation in DOA estimation," Journal of Systems Engineering and Electronics, vol. 19, no. 1, pp. 1-6, 2008.

[18] C. A. Balanis, Antenna Theory and Design, John Wiley \& Sons, New York, NY, USA, 2nd edition, 1997.

[19] J. Daniel, "Mutual coupling between antennas for emission or reception-application to passive and active dipoles," IEEE Transactions on Antennas and Propagation, vol. 22, no. 2, pp. 347-349, 1974.

[20] H. S. Lui, H. T. Hui, and M. S. Leong, "A note on the mutual coupling problems in transmitting and receiving antenna array," to appear in IIEEE Antennas and Propagation Magazine.

[21] T. K. Sarkar and O. Pereira, "Using the matrix pencil method to estimate the parameters of a sum of complex exponentials," IEEE Antennas and Propagation Magazine, vol. 37, no. 1, pp. 48-55, 1995.

[22] T. K. Sarkar, M. C. Wicks, M. Salazar-Palma, and R. J. Bonneau, Smart Antennas, John Wiley \& Sons, New York, NY, USA, 3rd edition, 2003.

[23] R. F. Harrington, Field Computation by Moment Methods, IEEE Press, New York, NY, USA, 1993.

[24] FEKO EM Software \& Systems S. A., (Pty) Ltd, 32 Techno Lane, Technopark, Stellenbosch, 7600, South Africa.

[25] H. S. Lui and H. T. Hui, "Effective mutual coupling compensation for direction-of-arrival estimations using a new, accurate determination method for the receiving mutual impedance," Journal of Electromagnetic Waves and Applications, vol. 24, no. 2-3, pp. 271-281, 2010.

[26] Y. Yu, H. T. Hui, and M. S. Leong, "Receiving mutual impedance of a compact patch antenna array with two elements in a rich multipath environment," in Proceedings of Asia Pacific Microwave Conference (APMC '09), Singapore, December 2009.

[27] W. M. Lee, Y. Yap, and H. T. Hui, "Receiving mutual impedance between two printed dipole antennas," Microwave and Optical Technology Letters, vol. 49, no. 4, pp. 892-896, 2007.

[28] Lui H. S., Y. Yu, H. T. Hui, and M. S. Leong, "Experimental study of mutual coupling compensation in direction finding using a compact antenna array," in Proceeding of the IEEE Asia Pacific Symposium on Electromagnetic Compatibilities, Beijing, China, April 2010.

[29] T. T. Zhang, Y. L. Lu, and H. T. Hui, "Compensation for the mutual coupling effect in uniform circular arrays for 2D DOA estimations employing the maximum likelihood technique," IEEE Transactions on Aerospace and Electronic Systems, vol. 44, no. 3, pp. 1215-1221, 2008.

[30] H. T. Hui, "An effective compensation method for the mutual coupling effect in phased arrays for magnetic resonance imaging," IEEE Transactions on Antennas and Propagation, vol. 53, no. 11, pp. 3576-3583, 2005.

[31] H. T. Hui, B. K. Li, and S. Crozier, "A new decoupling method for quadrature coils in magnetic resonance imaging," IEEE Transactions on Biomedical Engineering, vol. 53, no. 10, pp. 2114-2116, 2006.

[32] B. K. Li, H. T. Hui, C. H. Yang, and S. Crozier, "A new decoupling method for phased arrays in magnetic resonance imaging: an experimental approach," IEE Proceedings, Science, Measurement and Technology, vol. 2, no. 5, pp. 317-325, 2008.

[33] S. Lu, H. T. Hui, and M. Bialkowski, "Optimizing transmission strategy in single-user MIMO systems under the influence of antenna mutual coupling," IEEE Antennas And Wireless Propagation Letters, vol. 7, pp. 287-290, 2008.
[34] S. Lu, H. T. Hui, and M. Bialkowski, "Performance analysis of multiple-input multiple-output orthogonal frequency division multiplexing systems under the influence of antenna mutual coupling effect," IET Microwaves, Antennas and Propagation, vol. 3, no. 2, pp. 288-295, 2009. 

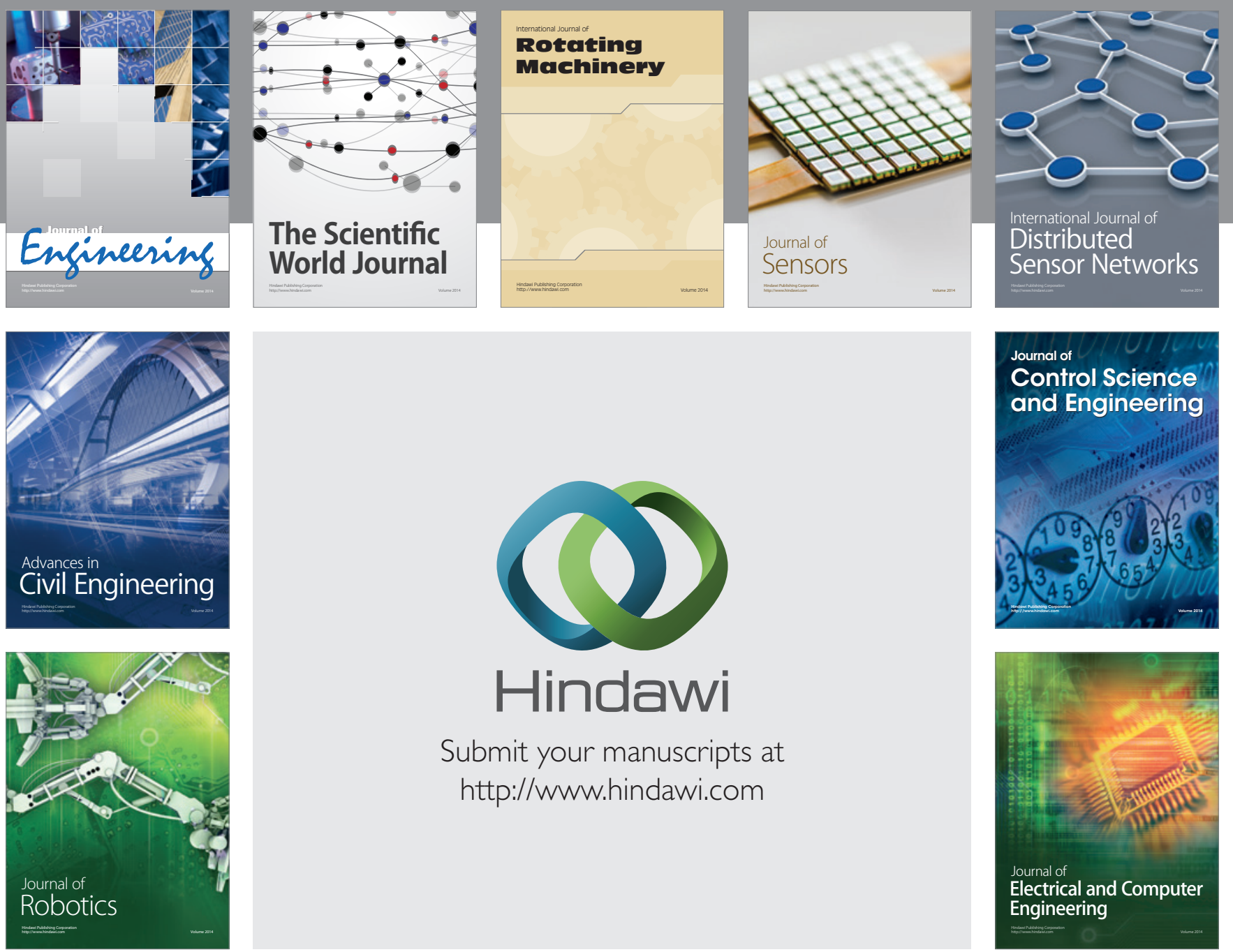

Submit your manuscripts at

http://www.hindawi.com
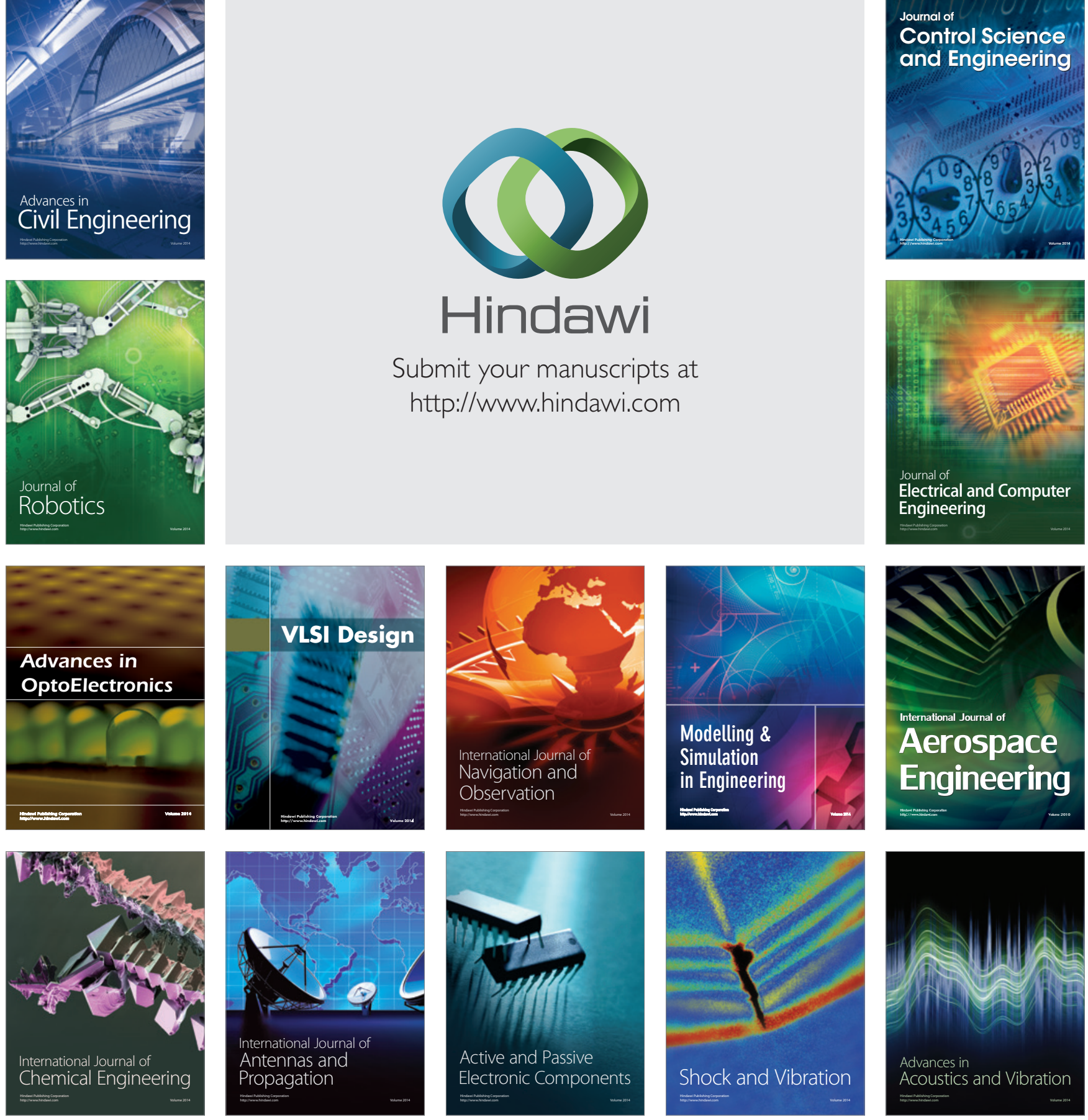